\title{
SOAP with self-reflection to improve student confidence regarding clinical reasoning competencies in community pharmacy practice
}

\author{
Kitiyot Yotsombut, Anuchai Theeraroungchaisri, Suntaree Watcharadamrongkun \\ Department of Social and Administrative Pharmacy, Faculty of Pharmaceutical Sciences, Chulalongkorn University, Thailand
}

Keywords
Clinical reasoning
Community pharmacy
Confidence
Reflection
SOAP note
Correspondence
Kitiyot Yotsombut
Department of Social and Administrative
Pharmacy
Faculty of Pharmaceutical Sciences
Chulalongkorn University
Thailand
Kitiyot.Y@pharm.chula.ac.th

\section{Introduction}

In order to provide an effective patient-centred pharmaceutical care, it is not only fundamental to memorise the information of the medications, but also to identify, understand, and solve the drug-related problems (DRPs), which involve effectiveness, safety, and adherence to the dispensed medications (Al-Quteimat \& Amer, 2016). The ability to ensure that dispensed medications are appropriate for individual patients and free from unnecessary DRPs requires a cognitive process, recognised as clinical reasoning (Croft et al., 2018). Clinical reasoning is a complex process that involves clinical cues collection, information processing, understanding of a patient's problems, needs, or situations, planning and implementing the interventions, evaluation outcomes, and reflecting or learning from the whole process of patient care (Linn et al., 2012). Therefore, clinical reasoning competencies (CRC) are not only vital for patient's clinical outcomes, but also for the development of clinical expertise for pharmacists.

The CRC should be gradually developed from the early years in pharmacy school, and fully established by graduation. Traditional classroom activities, lecturing and laboratory classes, may be insufficient to promote the 
students' CRC learning, since the CRC are highly complex and need authentic learning environments. For that reason, the experiential clerkships in community pharmacies, where they encounter real patients, is considered the main place for the CRC development in the pharmacy curriculum (Linn et al., 2012; Rencic, 2011). According to Kolb's cycle of experiential learning, attending the experiential clerkship creates the authentic situations that foster the CRC, such as the ability to collect clinical cues and implementing the interventions. However, certain components of the $\mathrm{CRC}$, which are more abstract, may not occur simultaneously but would occur after self-reflection. For example, students may have dispensed some clinically appropriate medications without truly understanding a patient's need at the time of the transaction. After they appraise the situation and their performance, they may improve their understanding of the patient's needs and find some mistakes. The lessons learned from selfreflection are, therefore, crucial for developing their own skills, knowledge, tactics, and other essential competencies (Maudsley \& Strivens, 2000).

Self-reflection is a self-evaluation process that leads to selfawareness of the students to their strengths and limitations. Following the self-reflection, self-development and self-confidence are substantially improved (Fry \& MacGregor, 2014; McBrien, 2007). Self-confidence has been shown to be highly correlated with engagement in the patient care process. High level of self-confidence in the CRC directly influences the ability of the clinician to perform their tasks appropriately and successfully. Selfconfidence also encourages the incorporation of CRC into routine clinical practice (Fry \& MacGregor, 2014; Martins et al., 2014).

In order to foster the CRC and self-confidence of pharmacy students when they are attending the clerkship in community pharmacies, the researchers have invented a $\mathrm{SOAP}^{+}$(called 'SOAP-PLUS') writing. The SOAP+ is a modified version of the subjective, objective, assessment, and plan (SOAP) note which is a well-known, problem-oriented documentation tool (Pearce et al., 2016). The SOAP+ has two columns: the left column is the standard SOAP note and the right column is the self-reflection. This format is specifically designed to facilitate student's self-evaluation and elaboration of their CRC step by step, from clinical data collection in ' $\mathrm{S}$ ' of the SOAP note to designing of patient care plan in ' $P$ '. The key points for self-reflection include completeness of relevant data, rationale of DRPs assessment, rationale of pharmacotherapeutic plan, appropriateness of monitoring plan, and appropriateness of communication skills. The SOAP+ writing has been implemented at the Faculty of Pharmaceutical Sciences, Chulalongkorn University, Thailand, since June 2016. The students were instructed to prepare two SOAP+ for each six-week clerkship and submit them to their preceptors and faculty staffs for assessment. Although the SOAP+ writing has been implemented for months, its impact on student confidence in the CRC as well as perceived benefits of the $\mathrm{SOAP}^{+}$have not been evaluated.

The aims of the current study were to explore the opinions of pharmacy students regarding their confidence in the CRC in community pharmacy practice following the SOAP+ writing and benefits of the reflection in $\mathrm{SOAP}^{+}$writing.

\section{Methods}

A self-administered questionnaire, developed by the researchers, was used to obtain the opinions of sixth year pharmacy students. The opinions regarding confidence in the CRC were self-rating scores ranging from 0 (lack of confidence in such competency) to 10 (absolute confidence). This part of the questionnaire consisted of 13 questions. The constructs of CRC in community pharmacy practice used in the questionnaire, were identified by thorough review of the position statement of Thai Community Pharmacy Association (Thai CPA) and validated by eight experts. The experts were instructors from 19 pharmacy schools who were nominated as the committee of the experiential learning in community pharmacy practice and have taught topics related to community pharmacy practice for more than five years. The final draft of the questionnaire was pre-tested on a sample of five sixth year students from other universities to ensure completeness of data capture and reduce ambiguity. This resulted in minor modifications of the final survey instrument. Semi-structured questions, guided by the survey results, were used in a focus group discussion.

\section{Data Collection}

There were seven clinical clerkships in community pharmacy in the 2016 academic year at Faculty of Pharmaceutical Sciences, Chulalongkorn University. Each clerkship lasted for six weeks. The first clerkship started in March 2016, and the seventh clerkship ended in March 2017. Data collection was conducted during the last week of June 2017 when all clinical clerkships were completed.

The sixth year pharmacy students were asked to complete the questionnaire. Of the 75 possible students: 54 students were available for quantitative survey, since 21 students attended the first or second clerkship and completed their clerkship before June 2016 when the SOAP+ writing was implemented. Subsequently, 16 out of 54 students were purposively approached, based on the completeness of their $\mathrm{SOAP}^{+}$writing and improvement of the self-rating 
scores on confidence in CRC after $\mathrm{SOAP}^{+}$writing. All approached students voluntarily joined the focus group discussion without deluding or intimidation.

Permission to conduct the study was approved from the Institutional Review Board at Chulalongkorn University (COA No. 127/2017). Informed consents were obtained before the data collection process.

\section{Data analysis}

In the current study, the authors compared the self-rating scores on confidence in CRC before SOAP note writing (Pre$S)$, after SOAP note writing without self-reflection (Post-S), and after the self-reflection writing to complete the SOAP+ (Post-R). Repeated-measure ANOVA was used and a-priori level of significance was set at 0.05 with Bonferroni adjustment. Descriptive statistics were reported for all survey items and participant demographics. All statistical analyses were conducted using SPSS 22.0. Contents from the focus group discussions were audiotaped with verbatim transcription. Thematic analysis of the transcribed text was conducted by two independent coders. Disagreements between the coders were discussed and solved by consensus.

\section{Results}

The sixth year students in this study were 25 males and 50 females (33.3\% and 66.7\%, respectively). 56 (74.7\%) students had attended one clinical clerkship in community pharmacy and $25.3 \%$ had two consecutive clinical clerkships in community pharmacy. Only 54 students, however, were eligible to be respondents of the questionnaires. 37 questionnaires were completed resulting in a $68.5 \%$ response rate. There were ten males (27.0\%) and 27 females (73.0\%). 30 (81.0\%) students had attended one clinical clerkship in community pharmacy and $19.0 \%$ had two consecutive clinical clerkships in community pharmacy. Of the 16 students who were informants on the focus group discussions $62.5 \%$ were male, and $56.3 \%$ of them had attended two consecutive clinical clerkships in community pharmacy.

Table I provides comparisons on mean scores of confidence in CRC before SOAP note writing (Pre-S), after SOAP note writing without self-reflection (Post-S), and after the self-reflection writing to complete the SOAP+ (Post-R). The statistically significant differences are found on all items $(p<0.05)$. The confidence in CRC was rated to be highest after students wrote the self-reflection (Post-R).

The results of post hoc analysis of self-rating scores on confidence in CRC are demonstrated in Table II. The mean differences between the Pre-S and Post-R ranged from 2.6 to 3.6 with statistically significant difference for all comparisons $(p<0.05)$. The mean differences between the Post-S and Post-R ranged from 0.2 to 1.2. The Post-S and Post- $R$ are statistically significant difference for most of CRC, except for the CRC\#2: using suitable clinical data related to the presenting problem, CRC\#8: planning for patient monitoring, and CRC\#10: utilisation of medical and pharmaceutical information resources. The CRC\#6: planning for pharmacologic therapy has the highest differences in both comparisons.

Three themes of perceived benefits of reflection in the $\mathrm{SOAP}^{+}$writing emerged through analysis of the transcripts:

1) Promotion of self-directed learning,

2) CRC improvement

3) Improvement of assessment validity. The themes and sub-themes are summarised in table III with specific quotes given in table IV.

The first theme, 'promoting self-directed learning', was illustrated by the fact that writing the reflection in the SOAP+ promotes learning from own experiences. It could stimulate the students to be aware of their strengths and limitations. As a result, they could successfully identify the knowledge or skills which should be improved. Some students had designed their own learning methods, such as self-questioning, which they perceived to be more efficacious than traditional classroom methods.

I asked myself about my thought and my knowledge when I wrote the reflection. Then I read to answer my questions and repeated the processes. I felt that it was enjoyable and more effective for me than lecturing.'

[Table IV, Student 2, Male]

The 'CRC improvement' emerged as another strong theme. The reflection could be helpful for students in reviewing and improving their CRC. This is because the reflection writing process promotes the systematic and critical thinking. In addition, the reflection writing process drives the students to review previous class contents and gain new knowledge or skills by self-directed learning. As the survey results show, student confidence in their CRC were substantially improved following reflection writing. The engagement in patient care could be improved by the reflection of the $\mathrm{SOAP}^{+}$as well.

'Since my thinking ability in drug dispensing has improved, my confidence in patient care is better, especially with the similar cases or situations.'

[Table IV, Student 3, Male] 
Table I: Comparisons of Self-Rating Score of Confidence in the CRC

\begin{tabular}{|c|c|c|c|c|}
\hline Clinical reasoning competency & $\begin{array}{l}\text { Mean (SD) of } \\
\text { Pre-S* score }\end{array}$ & $\begin{array}{c}\text { Mean (SD) } \\
\text { of Post-S** } \\
\text { score }\end{array}$ & $\begin{array}{c}\text { Mean (SD) } \\
\text { of Post-R*** } \\
\text { score }\end{array}$ & $p$ value \\
\hline CRC\#1: Collection of a patient's essential data & $5.4(1.7)$ & $7.9(1.3)$ & $8.67(1.0)$ & $<0.001$ \\
\hline CRC\#2: Using suitable clinical data related to the presenting problem & $5.7(1.9)$ & $8.1(1.2)$ & $8.4(1.1)$ & $<0.001$ \\
\hline CRC\#3: Problem identification and clinical diagnosis & $5.2(1.8)$ & $7.8(1.2)$ & $8.7(0.9)$ & $<0.001$ \\
\hline CRC\#4: Designing of suitable therapeutic & $5.7(1.8)$ & $8.0(1.4)$ & $9.0(0.8)$ & $<0.001$ \\
\hline CRC\#5: Planning for non-pharmacologic therapy & $5.5(1.9)$ & $8.0(1.4)$ & $8.7(1.0)$ & $<0.001$ \\
\hline CRC\#6: Planning for pharmacologic therapy & $5.5(1.9)$ & $8.0(1.3)$ & $9.1(0.8)$ & $<0.001$ \\
\hline CRC\#7: Planning for patient education and counselling & 6.0. (1.9) & $8.2(1.3)$ & $9.0(0.9)$ & $<0.001$ \\
\hline CRC\#8: Planning for patient monitoring & $5.7(1.9)$ & $8.2(1.3)$ & $8.6(1.0)$ & $<0.001$ \\
\hline CRC\#9: Designing a future plan & $5.4(1.7)$ & $8.0(1.4)$ & $8.7(1.0)$ & $<0.001$ \\
\hline CRC\#10: Utilisation of medical and pharmaceutical information resources & $5.9(2.0)$ & $8.4(1.1)$ & $8.7(1.0)$ & $<0.001$ \\
\hline CRC\#11: Clinical knowledge regarding the problem in the presenting patient & $5.9(2.1)$ & $8.0(1.2)$ & $8.5(1.0)$ & $<0.001$ \\
\hline CRC\#12: Gaining new skills and knowledge related to patient care & $5.8(1.9)$ & $8.2(1.2)$ & $8.8(0.9)$ & $<0.001$ \\
\hline CRC\#13: Systematic and critical thinking & $5.8(2.0)$ & $8.1(1.3)$ & $8.9(0.7)$ & $<0.001$ \\
\hline
\end{tabular}

*before SOAP note writing

**after SOAP note writing without self-reflection

***after the self-reflection writing to complete the SOAP+

\section{Table II: Post Hoc Analysis of Self-Rating Score of Confidence in the CRC}

\begin{tabular}{|c|c|c|c|}
\hline \multirow[t]{2}{*}{ Clinical reasoning competency } & \multicolumn{3}{|c|}{ Mean differences } \\
\hline & Pre-S+ vs Post-S++ & $\begin{array}{c}\text { Pre-S+ vs Post- } \\
\mathbf{R}^{+++}\end{array}$ & $\begin{array}{c}\text { Post-S }{ }^{++} \text {vS } \\
\text { Post-R }{ }^{+++}\end{array}$ \\
\hline CRC\#1: Collection of a patient's essential data & $2.5^{*}$ & $3.2^{*}$ & $0.7^{*}$ \\
\hline CRC\#2: Using suitable clinical data related to the presenting problem & $2.4^{*}$ & $2.7^{*}$ & $0.3\left(\mathrm{NS}^{* *}\right)$ \\
\hline CRC\#3: Problem identification and clinical diagnosis & $2.7^{*}$ & $3.5^{*}$ & $0.9 *$ \\
\hline CRC\#4: Designing of suitable therapeutic goals & $2.4^{*}$ & $3.3^{*}$ & $1.0^{*}$ \\
\hline CRC\#5: Planning for non-pharmacologic therapy & $2.5^{*}$ & $3.2^{*}$ & $0.7^{*}$ \\
\hline CRC\#6: Planning for pharmacologic therapy & $2.4^{*}$ & $3.6^{*}$ & $1.2^{*}$ \\
\hline CRC\#7: Planning for patient education and counselling & $2.2^{*}$ & $3.0^{*}$ & $0.8^{*}$ \\
\hline CRC\#8: Planning for patient monitoring & $2.5^{*}$ & $2.9^{*}$ & $0.4\left(\mathrm{NS}^{* *}\right)$ \\
\hline CRC\#9: Designing a future plan & $2.7^{*}$ & $3.4^{*}$ & $0.7^{*}$ \\
\hline CRC\#10: Utilisation of medical and pharmaceutical information resources & $2.5^{*}$ & $2.7^{*}$ & $0.2\left(\mathrm{NS}^{* *}\right)$ \\
\hline CRC\#11: Clinical knowledge regarding the problem in the presenting patient & $2.1^{*}$ & $2.6^{*}$ & $0.5^{*}$ \\
\hline CRC\#12: Gaining new skills and knowledge related to patient care & $2.4^{*}$ & $3.0^{*}$ & $0.6^{*}$ \\
\hline CRC\#13: Systematic and critical thinking & $2.3^{*}$ & $3.1^{*}$ & $0.8^{*}$ \\
\hline
\end{tabular}

* the mean difference is significant at the 0.05 level

** non-significant difference

+before SOAP note writing

++after SOAP note writing without self-reflection

${ }^{+++}$after the self-reflection writing to complete the SOAP+ 
Table III: Themes and Sub-themes Identified from the Focus Group Discussions of Pharmacy Students

\begin{tabular}{ll}
\hline Theme & Sub-themes \\
\hline Promotion of self-directed learning & Learning from experiences \\
& Self-awareness \\
& Self-directed learning \\
& Self-motivated learning \\
CRC improvement & Improved systematic and logical thinking \\
& Improved clinical skills and knowledge \\
& Improved self-confidence \\
Improvement of assessment validity & Increased patient care engagement \\
\hline
\end{tabular}

Table IV: Key Themes with Supporting Quotes from Focus Group Participants

\begin{tabular}{|c|c|}
\hline Theme & Quotes \\
\hline Promotion of self-directed learning & $\begin{array}{l}\text { 'I had reviewed my thought, my skills, and my performances when I wrote the SOAP+. Therefore, I } \\
\text { learnt which are work and not work. I, now, know what should be better for the next patient } \\
\text { care' [Student 3, Male]. } \\
\text { 'I think the SOAP+ is a good tool for self-directed leaning because when I reflect on my dispensing } \\
\text { practice, I was aware of my mistakes. Therefore, I can tell myself what knowledge should be } \\
\text { studied or what skill should be improved' [Student 11, Female]. } \\
\text { 'Even I have just finished SOAP writing, I had to search and read more articles again. It was } \\
\text { because I realized that I missed something when I wrote the reflection' [Student 14, Male]. } \\
\text { 'After reflection, I was aware that my nervousness is not caused by unfamiliar practice } \\
\text { environment, but because I was not confident about that disease. I prepared myself about the } \\
\text { disease that night. I performed better on the next day' [Student } 13 \text {, Male]. } \\
\text { 'I asked myself about my thought and my knowledge when I wrote the reflection. Then I read to } \\
\text { answer my questions and repeated the processes. I felt that it was enjoyable and more } \\
\text { effective for me than lecturing' [Student 2, Male]. }\end{array}$ \\
\hline
\end{tabular}

CRC improvement

Improvement of assessment validity
I had to asked myself about the rationale of my dispensing practice when I wrote the reflection. My thinking process was more systematic and logical after finishing the SOAP+' [Student 5, Female].

'I had to read more when I wrote the SOAP+. I have just understood some topics that were taught in class in the last few years. Besides, some topics were never taught or mentioned in classroom. Therefore, my clinical knowledge is better because of this assignment (the SOAP+)' [Student 14, Male].

'My SOAP+ was about intimate problem. I felt reluctant to interview the patient and missed important information. After the reflection, I got an excellent technique for interviewing about that organ' [Student 13, Male].

'Sometimes, my regimen was different from my friends. When I wrote the reflection, I had to evaluate my regimen thoroughly and compare with others. I found that both regimens had comparable efficacy and were appropriate for different situations. Therefore, I had learnt new thing and did not lose my confidence in patient care' [Student 6, Female].

'Since my thinking ability in drug dispensing has improved, my confidence in patient care is better, especially with the similar cases or situations' [Student 3, Male].

'I had tried my new technique for patient interviewing and found it was useful. I got it when I wrote the reflection. I felt more confident and engaged in caring for people' [Student 13, Male].

'I and my friends were aware of our mistakes by the reflection. The quality of patient service by us might be improved and reoccurrence of medication errors could be prevented' [Student 12, Male].

'The SOAP+ helped me communicate with my preceptor and faculty staff because my reflection was about my logical thinking. I believe that they can understand me more than just reading my regular SOAP note' [Student 9, Male].

'I had some concerns about drug selection for my patient, but I did not write them in my SOAP because they did not fit the format of the SOAP note. I like the reflection for that reason' [Student 8, Male].

'Writing the reflection encouraged me to review and prepare for the presentation. I presented my case with confidence and conveyed my thought successfully' [Student 1. Male]. 
The theme 'improvement of assessment validity' was unexpectedly identified. The SOAP note has been used as a tool for student assessment by the Faculty of Pharmaceutical Sciences, Chulalongkorn University, for a long time. Some components of the CRC could not be demonstrated clearly due to the traditional and limiting format of the SOAP note. As a result, the student assessment for CRC would not be accurate. Incorporating the self-reflection into the SOAP note is a good opportunity for students to explain their rationale, concern, and thinking process. The reflection writing in the $\mathrm{SOAP}^{+}$also improves the readiness of students in case presentation at the practice sites. Understanding between students and faculty staff are reconciled and the assessment validity of the assessment is increased.

'The SOAP' helped me communicate with my preceptor and faculty staff because my reflection was about my logical thinking. I believe that they can understand me more than just reading my regular SOAP note'

[Table IV, Student 9, Male]

\section{Discussion}

This mixed method study provides insight into pharmacy students' views on incorporating self-reflection into the SOAP note (the SOAP+) and their confidence regarding CRC in community pharmacy. The students in this study reported that their confidence in most constructs of CRC were statistically significantly improved following SOAP+ writing, when compared with SOAP writing without selfreflection. The finding of the study is consistent with other studies which have demonstrated the importance of reflective practice on development of professional skills, attitudes, and self-confidence in clinical skills and knowledge (Chaffey, de Leeuw, \& Finnigan, 2012; King \& Bithell, 1998; Lutz et al., 2013; Mann, Gordon, \& MacLeod, 2007; Tsingos, Bosnic-Anticevich, \& Smith, 2014).

Self-confidence has been shown to be a critical factor for reaching desired clinical outcomes. Improved quality of patient care was more commonly experienced by health care providers who perceived a higher degree of selfconfidence in clinical competency, such as patient interviewing with proper communication technique and understanding of a patients' needs (Hecimovich \& Volet, 2009; Mazzo et al., 2015). In addition, self-confidence has been shown to be a strong predictor for success in professional roles, job satisfaction, and career longevity (Judge et al., 2007; Stajkovic \& Luthans, 1998). Medical students who had low degrees of self-confidence also reported to have higher feelings of stress and anxiety, which negatively affected their cognitive capability and CRC. Therefore, reluctant, inappropriate, or harmful clinical judgment may occur when students have low selfconfidence. Self-reflection has been found to be an effective tool to prevent such problems since it reduces stress, improves patient care, enhances personal and professional competency development, and leads to improved self-confidence of the student (Lutz et al., 2013). Improved self-confidence in $\mathrm{CRC}$ through $\mathrm{SOAP}^{+}$writing is, therefore, useful for pharmacy students and should continue to be implemented in the pharmacy curriculum.

The Post-Hoc analysis for confidence in the CRC found that the confidence in using suitable clinical data related to the presenting problem (CRC\#2), planning for patient monitoring (CRC\#8), and utilisation of medical and pharmaceutical information resources (CRC\#10) were not significantly improved following $\mathrm{SOAP}^{+}$when compared with SOAP writing without self-reflection. The CRC\#2 and CRC\#10 involved the use of data and information resources which might be ultimately improved when students write their SOAP note, rather than reflection (Pearce et al., 2016). These CRCs had relatively high Post-S with noticeable differences between Pre-S and Post $\mathrm{S}$. The patient monitoring in community pharmacies in Thailand has not been achieved in practice. The concrete outcome of the CRC\#8 could not be observed. As a result, the self-reflection might not remarkably improve the confidence in this CRC. Although unimproved confidence scores may not necessarily imply that the students have low level of competencies, the CRCs are considered vital for effective patient care. Further study is needed to understand and resolve this issue.

The usefulness of the self-reflection in the SOAP+ is confirmed in our qualitative thematic analysis includes promoting of self-directed learning, CRC improvement, and improvement of assessment validity. From the authors observations and interviews with the students, cases that contains drug-related problems (DRPs), medication errors (MEs), or complicated issues, were more likely to be selected for the SOAP+ writing. Therefore, mistakes, lack of confidence, knowledge and skill inadequacy, or unexpected outcomes were inevitably common. Owing to the sophisticated characteristics of cases chosen, self-directed learning and the CRC of students in the study could be improved as explained by the Kolb's cycle of experiential learning (Fernandez, Chelliah, \& Halim, 2015; Tsingos et al., 2014).

There are some limitations in this study which should be considered when using these findings. This was a pilot study with a small number of students from a single 
institution. Although the proportion of gender and number of clinical clerkships in community pharmacy of the questionnaire respondents were similar to the whole class of sixth year students, the informants of the qualitative analysis were not the same, with a higher proportion of male students with two-consecutive clinical clerkships. All the approached students had experienced previous clinical clerkships which could improve the CRC before their community clerkships. The generalisability of these finding, therefore, may be limited. Nine students (56.3\% of the qualitative informants) attended two consecutive clinical clerkships in community pharmacy. These students had more experience with the SOAP+ and may have different opinions. However, the subgroup analysis from a number of clinical clerkships did not found any significant differences. The data collection at the end of the academic year is also a limitation of this study since there is risk of recall bias. Further study with a larger number of students from various institutions is warranted and should be designed to reduce recall bias of the students.

\section{Conclusions}

The study demonstrates that $\mathrm{SOAP}^{+}$, a novel version of SOAP note, improves student confidence regarding the CRC in community pharmacy practice. These findings may result from the self-reflection in $\mathrm{SOAP}^{+}$that has been shown to promote self-evaluation, self-awareness, and improve self-confidence. Improved self-confidence, especially in CRC, has positive impact on student performance and patient clinical outcomes. Therefore, the $\mathrm{SOAP}^{+}$could be an effective tool for pharmacy students attending clinical clerkship in community pharmacies.

\section{Acknowledgments}

The authors wish to thank Professor Jeanine K. Mount from Massachusetts College of Pharmacy and Health Sciences, in the United States of America, and Associate Professor Sathitpong Thanaviriyakul from the Faculty of Pharmaceutical Sciences, Chulalongkorn University for their collaboration and encouragement with this study.

\section{Funding disclosure}

None

\section{Conflicts of Interest}

None

\section{References}

Al-Quteimat, O.M., \& Amer, A.M. (2016). Evidence-based pharmaceutical care: the next chapter in pharmacy practice. Saudi Pharmaceutical Journal, 24(4), 447-451. https://doi.org/10.1016/ j.jsps.2014.07.010

Chaffey, L., de Leeuw, E.J., \& Finnigan, G. (2012). Facilitating students' reflective practice in a medical course: literature review. Education for Health, 25(3), 198-203. https://doi.org/ $\underline{10.4103 / 1357-6283.109787}$

Croft, H., Gilligan, C., Rasiah, R., Levett-Jones, T., \& Schneider, J. (2018). Thinking in pharmacy practice: a study of community pharmacists' clinical reasoning in medication supply using the think-aloud Method. Pharmacy, 6(1), 1. https://doi.org/10.3390/ pharmacy 6010001

Fernandez, S.C., Chelliah, K.K., \& Halim, L. (2015). A peek into oneself: reflective writing amongst undergraduate medical imaging students. Reflective Practice, 16(1), 109-122. https:// doi.org/10.1080/14623943.2014.982524

Fry, M., \& MacGregor, C. (2014). Confidence and impact on clinical decision-making and behaviour in the emergency department. Australasian Emergency Nursing Journal, 17(3), 91-97. https:// doi.org/10.1016/i.aenj.2014.03.003

Maudsley, G., \& Strivens, J. (2000). Promoting professional knowledge, experiential learning and critical thinking for medical students. Medical Education, 34(7), 535-544. https://doi.org/ $\underline{10.1046 / \mathrm{i} .1365-2923.2000 .00632 . x}$

Hecimovich, M.D., \& Volet, S.E. (2009). Importance of building confidence in patient communication and clinical skills among chiropractic students. Journal of Chiropractic Education, 23(2), 151-164. https://doi.org/10.7899/1042-5055-23.2.151

Judge, T.A., Jackson, C.L., Shaw, J.C., Scott, B.A., \& Rich, B.L. (2007). Self-efficacy and work-related performance: the integral role of individual differences. Journal of applied psychology, 92(1), 107. https://doi.org/10.1037/0021-9010.92.1.107

King, M.C.A., \& Bithell, M.C. (1998). Expertise in diagnostic reasoning: a comparative study. British Journal of Therapy and Rehabilitation, 5(2), 78-87. https://doi.org/10.12968/ bjtr.1998.5.2.14219

Linn, A., Khaw, C., Kildea, H., \& Tonkin, A. (2012). Clinical reasoning - a guide to improving teaching and practice. Australian Family Physician, 41(1-2), 18-20.

Lutz, G., Scheffer, C., Edelhaeuser, F., Tauschel, D., \& Neumann, M. (2013). A reflective practice intervention for professional development, reduced stress and improved patient care--a qualitative developmental evaluation. Patient Education and Counseling, 92(3), 337-345. https://doi.org/10.1016/..pec.2013.03.020

Mann, K., Gordon, J., \& MacLeod, A. (2007). Reflection and reflective practice in health professions education: a systematic review. Advances in Health Sciences Education, 14(4), 595. https:// doi.org/10.1007/s10459-007-9090-2.

Martins, J.C.A., Baptista, R.C.N., Coutinho, V.R.D., Mazzo, A., Rodrigues, M.A., \& Mendes, I.A.C. (2014). Self-confidence for emergency intervention: adaptation and cultural validation of the self-confidence scale in nursing students. Revista Latino-Americana de Enfermagem, 22(4), 554-561. https://doi.org/ $\underline{10.1590 / 0104-1169.3128 .2451}$ 
Mazzo, A., Martins, J.C.A., Jorge, B.M., Batista, R.C.N., Almeida, R.G.d.S., Henriques, F.M.D., Coutinho, V.R.D., \& Mendes, I.A.C. (2015). Validation of the self-confidence scale of nursing care in urinary retention. Revista Latino-Americana de Enfermagem, 23, 814-820. https://doi.org/10.1590/0104-1169.0256.2619

McBrien, B. (2007). Learning from practice--reflections on a critical incident. Accident and Emergency Nursing, 15(3), 128-133. https://doi.org/10.1016/i.aaen.2007.03.004

Pearce, P.F., Ferguson, L.A., George, G.S., \& Langford, C.A. (2016). The essential SOAP note in an EHR age. Nurse Practitioner, 41(2), 29-36. https://doi.org/10.1097/01.NPR.0000476377.35114.d7

Rencic, J. (2011). Twelve tips for teaching expertise in clinical reasoning. Medical Teacher, 33(11), 887-892. https://doi.org/ $\underline{10.3109 / 0142159 X .2011 .558142}$

Stajkovic, A.D., \& Luthans, F. (1998). Self-efficacy and work-related performance: a meta-analysis. Psychological bulletin, 124(2), 240. https://doi.org/10.1037/0033-2909.124.2.240

Tsingos, C., Bosnic-Anticevich, S., \& Smith, L. (2014). Reflective practice and its implications for pharmacy education. American Journal of Pharmaceutical Education, 78(1), 18. https://doi.org/ $\underline{10.5688 / a j p e 78118}$ 